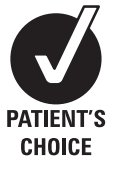

An additional table is published online only at http:// jnnp.bmi.com/content/vol79/ issue 10

${ }^{1}$ Helen Durham Neuroinflammatory Centre Department of Neurology, University Hospital of Wales Heath Park, Cardiff CF14 4XN, UK; ${ }^{2}$ Department of Neurology, Ninewells Hospital, Dundee DD1 9SY, UK; ${ }^{3}$ Department of Clinical Neurosciences University of Cambridge Clinical School, Addenbrooke's Hospital, Cambridge CB2 000, UK

Correspondence to: N Robertson, Helen Durham Neuro-inflammatory Centre, Department of Neurology, University Hospital of Wales Heath Park, Cardiff CF14 4XN UK; robertsonnp@cardiff.ac.uk

Received 31 August 2007 Revised 7 February 2008

Accepted 7 February 2008

Published Online First

26 February 2008

\title{
Change in disability in patients with multiple sclerosis: a 20 -year prospective population-based analysis
}

\author{
C Hirst, ${ }^{1}$ G Ingram, ${ }^{1}$ R Swingler, ${ }^{2}$ D A S Compston, ${ }^{3}$ T Pickersgill, ${ }^{1}$ N P Robertson ${ }^{1}$
}

\begin{abstract}
Background: In patients with multiple sclerosis (MS), the natural history of the disease is of considerable importance to predict and understand long-term outcome and inform choices made by patients and clinicians. This information should ideally be derived from data that reflects the entire disease course.

Methods: In this study, morbidity data from a prevalent cohort established in 1985 have been re-examined after an interval of 20 years to assess factors that may be important in determining outcome.
\end{abstract}

Results: Of 379 patients who fulfilled criteria for definite or probable MS in the original population-based cohort, 221 (58.3\%) had died, 149 (39.3\%) were alive and 9 (2.4\%) were untraceable. Mean Expanded Disability Status Scale (EDSS) score in 1985 was 5.15 (SD 2.7, range $0-9.5$ ) and 8.01 (SD 2.6, range $0-10$ ) in those alive in 2005. Mean worsening of EDSS scores in surviving patients was +3.02 EDSS points, but $14.0 \%$ had worsened by $<1$ EDSS point over 20 years. $61.4 \%$ of patients with EDSS $3.5-5.5$ and $82.2 \%$ of those with an EDSS of $\leqslant 3$ in 1985 had an EDSS of $\geqslant 6$ after 20 years. Lower baseline EDSS scores $(p<0.0001)$, higher pyramidal functional system score $(p=0.02)$ and a greater number of functional systems involved $(p=0.001)$ were significantly more likely to be associated with greater worsening of disability. Of those with benign disease in 1985 , only $19 \%$ remained benign after 20 years of followup; however, $12.6 \%$ of patients had minimal disability after at least 20 years after their disease onset and 14\% of patients failed to worsen by $\geqslant 1$ EDSS point.

Conclusions: This study emphasises the importance of long-term epidemiological studies and the development of clinically relevant measures that effectively predict outcome and can guide decisions on therapeutic management.

Multiple sclerosis (MS) is characteristically a neurological disease of considerable prognostic variability. The natural history ranges from a disorder exhibiting a mildly relapsing disease course with episodes separated by many decades without significant accumulation of disability, to a syndrome of rapidly evolving disability and death within a few years. In a disease that has a median duration of $30-40$ years, ${ }^{1-4}$ it is of considerable importance both to predict and understand the long-term outcome so that informed choices can be made by patients and clinicians derived from information that reflects the entire disease course and allows shared decisions on disease management.

To gain an accurate understanding of changes in disability over the course of the disease, long-term analyses of unselected cohorts are required. To this end, a number of natural history studies have been undertaken. ${ }^{15-20}$ Few studies have been undertaken in the UK; ${ }^{10}{ }^{11-24}$ many were of relatively short duration; and adequate statistical methods accommodating censored data were not used in the earlier studies. ${ }^{21} 2526$ In addition, although many used population-based cohorts, ${ }^{10} 13162728$ a proportion were restricted to the examination of selected populations such as hospital-based cohorts, ${ }^{3}{ }^{29-34}$ which may have been biased towards more severe cases. ${ }^{2}$ Latterly, the introduction of a number of therapeutic interventions aimed at modifying the disease course have made it progressively more difficult to perform true contemporary natural history studies, which are unaffected by improvements in rehabilitation and symptom control. However, with the recent expansion in the type and number of these therapies, the need to identify patients with poor prognostic features is becoming increasingly important in order to guide early treatment decisions and/or consideration of interventions that may carry an unfavourable risk/ benefit profile. Conversely, identifying patients with a benign disease course should allow the avoidance both of potential side effects and the inconvenience of administering therapy, whilst also restricting unnecessary expenditure, which may in turn allow the allocation of resources to those patients for whom greatest benefit will be derived.

In this study, a population-based cohort established in 1985 and without significant access to disease-modifying treatments has been re-examined after an interval of 20 years to determine outcome and change in disability, and to assess factors that may be important in determining outcome. We have also determined the fate of those initially considered to have benign disease, as well as the group not fulfilling the Poser criteria at prevalence and so classified as "suspected" but would have been considered to have MS by earlier diagnostic criteria.

\section{METHODS}

A comprehensive population-based survey was performed in South Glamorgan, UK, in 1985, identifying patients with MS from general practice notifications, hospital episode statistics, a departmental index, the Multiple Sclerosis Society, and community nurses and physiotherapists. Patients were included if they were resident within the county of South Glamorgan, alive on prevalence day and fulfilled diagnostic criteria for MS. This 
study demonstrated a prevalence of $441 / 376718\left(117 / 10^{5}\right)$ on 1 January 1985 , with $86 \%$ of patients having clinically definite or probable MS according to the Poser criteria. ${ }^{36}$ Fourteen percent of patients were classified as having suspected MS and this group included patients who would previously have been classified as having MS using earlier diagnostic criteria of Allison and Millar, but did not fulfil the Poser criteria for definite or probable disease. The age range of prevalent patients was 10-85 years (mean, 48.7 years), mean age-at-onset was 32.2 years and mean age-at-diagnosis was 36.4 years. Data were collected on demographic characteristics, symptoms and time of disease onset, diagnosis and disability scores (including EDSS) from 301 of 441 prevalent patients obtained at interview and examination using standardised proformas. Of those with definite or probable MS, Expanded Disability Status Scale (EDSS) scores were obtained in 289 of 379 patients, with the remainder refusing or unable to take part in the study. A total of 339/441 (99.7\%) patients had never received disease-modifying therapies (DMTs).

All cases were flagged with the Office of Population Censuses and Surveys (OPCS), and copies of death certificates were collected prospectively. Those patients in whom no death certificate had been received were traced to ensure current vital status. After an interval of 20 years, a further epidemiological analysis was undertaken in an attempt to determine the fate of the original 441 patients. Patients alive on 1 January 2005 were traced. The hospital patient administration system (HPAS) was used to determine the last known address of the patient, and their General Practitioner (GP) was contacted to confirm residence. For patients not identified by the HPAS, the National Health Service Administrative Register (NHSAR), a database holding demographic data for residents registered with a local GP, was used to trace patients still resident in Wales. Information held in this database includes NHS number, name, address, date of birth, date of death and GP registration details. Following confirmation of identification and contact details and consent of the GP or treating consultant, patients were approached by letter either from their GP or directly from the Department of Neurology if still under regular review, and invited to take part in the study. After sending one reminder, those who agreed to participate were asked to attend a clinic appointment in the University Hospital of Wales or, alternatively, a domiciliary visit was undertaken, at which time a full EDSS score was established after neurological examination, and routine clinical and demographic information updated according to a standardised proforma. Morbidity data collected in 1985 were compared to current disability assessments to determine change over the 20-year period.

\section{Statistical methods}

Median observed survival was calculated using a Kaplan-Meier analysis. This is an established method of estimating time-toevent models in the presence of censored cases. It is based on estimating conditional probabilities at each time point when an event occurs and taking the product limit of those probabilities to estimate the survival rate at each point in time. In this study, the recorded event was death, and median survival was calculated by estimating the area under the cumulative survival curve derived from the original 1985 cohort. Median observed survival was therefore defined as the length of time of observation following disease onset at which half of the cohort were estimated to have reached the end point of death. Expected survival was defined as the median age at death calculated from published Welsh life tables ${ }^{37}$ for an equivalent hypothetical general population cohort matched for age and sex to year of disease onset of the cases.

Mean change in EDSS scores over 20 years was analysed by baseline factors, including present age, age-at-onset, sex, initial symptoms at disease onset, mono- verses poly-symptomatic onset, disease duration in 1985 and baseline EDSS score. An analysis of covariance model for the factors listed was used to assess change in EDSS score over 20 years, controlling for baseline EDSS score and disease duration in 1985. A multiple linear regression was also performed for the same variables, again controlling for baseline EDSS score and disease duration in 1985. Baseline characteristics of patients with and without an EDSS score recorded in 1985 were compared by $t$-test and the Chi-squared statistic. Patients who were known to have died of MS-related causes were assigned an EDSS score of 10.

\section{RESULTS}

\section{Case ascertainment and fate of all patients}

Of 441 patients identified in the original prevalence study, 380 had definite or probable disease according to the Poser criteria in 1985. By 2005, 10/380 were considered not to have MS. In each instance, a consultant neurologist had reviewed the patient and determined that an alternative diagnosis was more likely or post-mortem examination had been performed and no pathological evidence of MS revealed. In addition, a further 8 of 61 (13\%) patients with suspected MS in 1985 had the diagnosis of MS revised by a consultant neurologist who had reviewed the patient and felt that an alternative diagnosis was the cause of the patients signs and/or symptoms and that MS was not the cause: of the remainder, 18 (30\%) never had a second inflammatory event; 9 (15\%) did subsequently fulfill criteria for definite or probable MS; and 26 (43\%) could not be accurately determined (fig 1).

Alternative diagnoses for patients considered to have definite or probable disease in 1985 included posterior fossa meningioma, acute disseminated encephalomyelitis and hereditary spastic paraparesis; and in those with suspected MS included scleroderma, functional illness and multiple cavernomas. For all alternative diagnoses, see online table.

\section{Mortality}

Of the 379 patients who fulfilled criteria for definite or probable MS in 2005, 221 (58.3\%) had died, 149 (39.3\%) were alive and 9 $(2.4 \%)$ were untraceable. Mean age-at-death was 65.3 years (95\% confidence interval 63.4 to 67.1 , range 25.0 to 101.0 , SD 14.3). In those surviving to 2005 , there were 93 women $(75.6 \%)$ and 30 men $(24.4 \%)$. The mean age of patients was 61.3 years (range 39-82) and only $2(1.6 \%)$ had ever received DMTs. Median observed survival for the entire cohort was 37.6 years. The expected median survival for the entire cohort was 46.4 years. The crude death rate was 38.6/1000/year.

\section{Disability}

EDSS scores from 1985 were available in 289 patients with clinically definite or probable MS, of whom 123 were alive. No EDSS score was determined in 20 who were either lost to follow-up or declined to take part. 90 patients from 1985 had declined to take part in the study and therefore did not have EDSS scores recorded.

Mean EDSS score in 1985 was 5.15 (SD 2.7, range 0-9.5) and 8.01 (SD 2.6, range 0-10) in those alive in 2005. Mean worsening of those patients surviving and with EDSS scores recorded both in 1985 and 2005 was +3.02 EDSS points 
Figure 1 Case ascertainment and fate of all patients from 1985 prevalence study: definite and probable cases $(A)$ and suspected cases (B). MS, multiple sclerosis.

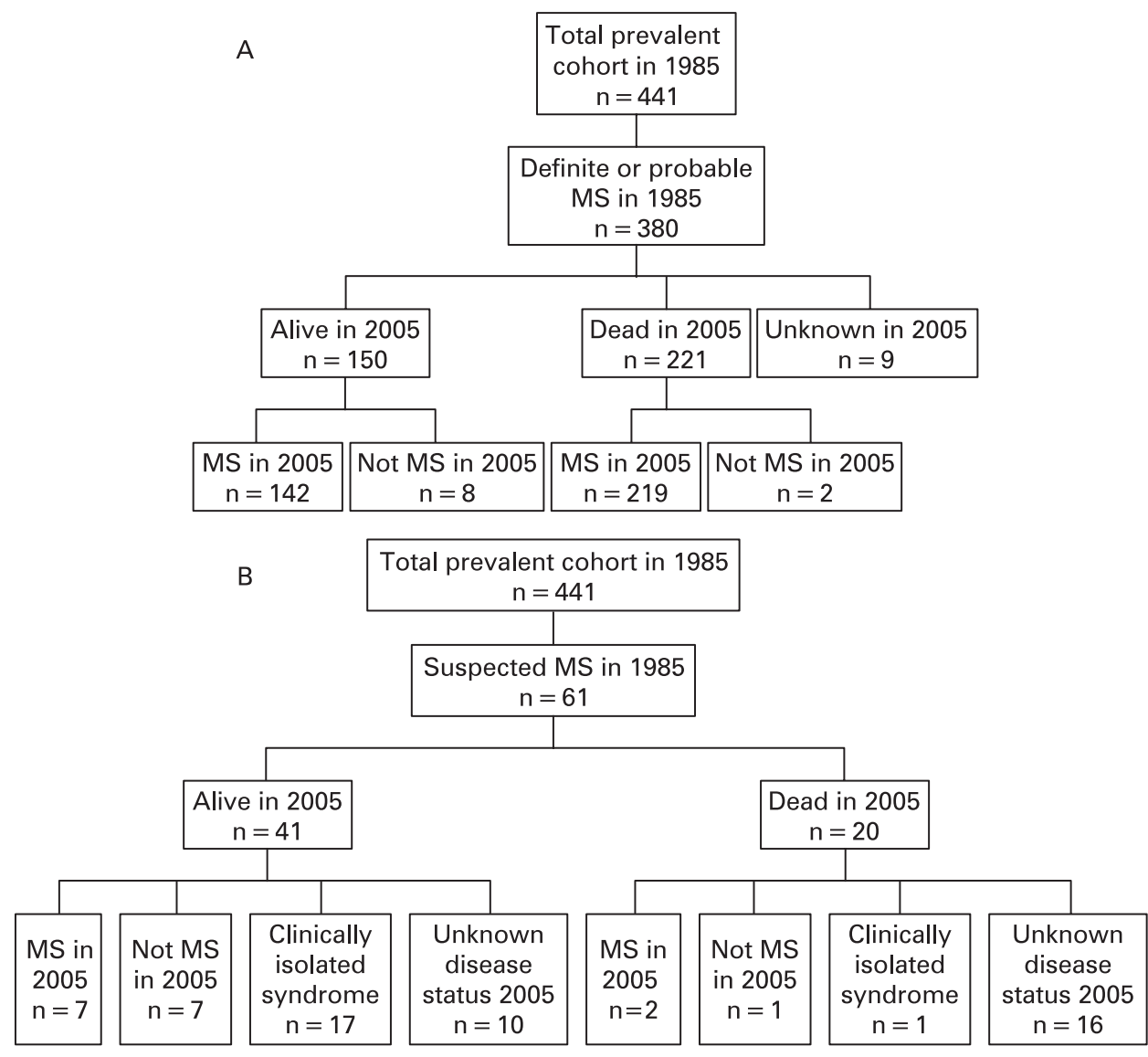

$(n=103)$. Total change in EDSS points for all patients, including those who died of MS-related causes over the study ( $n=199)$, was 573.5 points in 173 patients over a total of 2806.2 patient-years of follow-up, providing a mean change of +4.1 EDSS points per patient over 20 completed years of follow-up. Median duration from 1985 to death in those dying from MSrelated causes was 10.6 years.

Figure 2 shows the distribution of change in EDSS scores over 20 years. Of 199 patients with EDSS scores in 1985 and 2005,

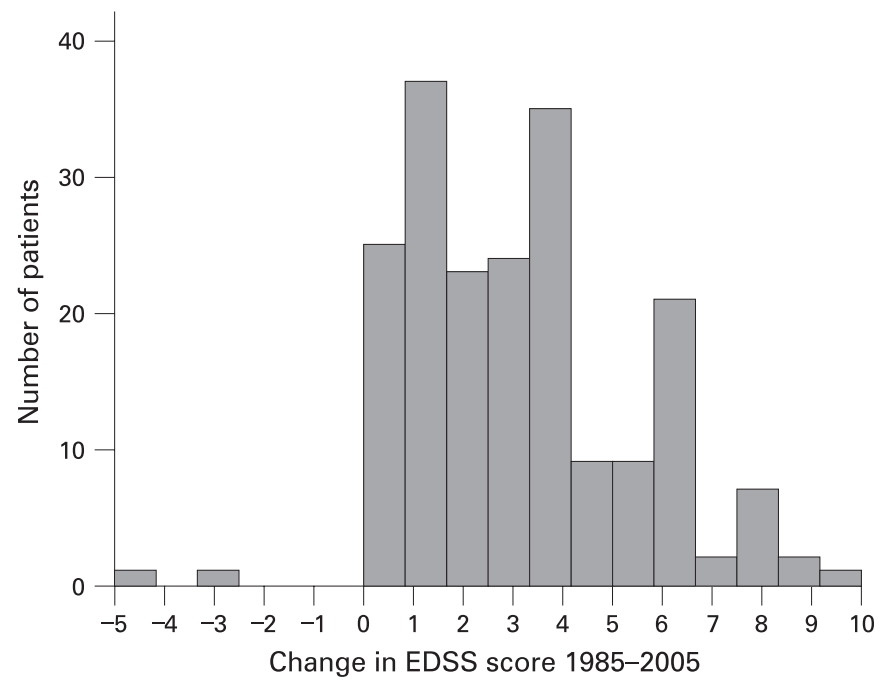

Figure 2 Change in Expanded Disability Status Scale (EDSS) scores between 1985 and 2005.
$171(85.9 \%)$ had worsened by $\geqslant 1$ EDSS point, 133 (66.8\%) by $\geqslant 2$ points, $95(47.7 \%)$ by $\geqslant 3$ points, $67(33.7 \%)$ by $\geqslant 4$ points and $42(21.1 \%)$ by $\geqslant 5$ points. Twenty-eight $(14.0 \%)$ had worsened by $<1$ EDSS point over 20 years, suggesting that this group had exhibited a stable disease course.

A total of $82.2 \%$ of patients with an EDSS score of 3.5-5.5 and $61.4 \%$ of those with an EDSS score of $\leqslant 3$ in 1985 required a stick to mobilise themselves or were more disabled for walking after 20 years (EDSS $\geqslant 6$ ). At least $37.1 \%$ of those with an EDSS score of $\leqslant 3$ in 1985 and $58.9 \%$ of those with an EDSS score of 3.5-5.5 became wheelchair users or bed-bound over the same time interval. Table 1 shows the change in EDSS scores between 1985 and 2005. Over 20 years, $16.2 \%$ of patients who required a minimum of unilateral support but were still mobile (EDSS 6-7.5) in 1985 had become wheelchair users or bed-bound; $36.5 \%$ had died of MSrelated causes and a further $31.1 \%$ had died of unrelated causes by 2005. By comparison, only $8.3 \%$ of those that were wheelchair users or bed-bound in 1985 were still alive in 2005 .

The impact of clinical variables recorded on mean EDSS change was examined (table 2). There was a trend for those who were younger in 1985 to have a greater worsening of EDSS score; however, this did not reach statistical significance. Sex, site of initial lesion or mono- compared to poly-regional onset had no impact on the degree of change in EDSS score.

The only significant factors affecting the mean change in EDSS score were disease duration in 1985 and EDSS score, with those having lower EDSS scores and shorter disease duration showing a greater mean change in EDSS score. Patients with disease duration $<10$ years at prevalence day 1985 had a mean EDSS change of +3.62 compared to +2.71 and +2.54 for those with disease durations of $10-25$ and $>25$ years, respectively. 
Table 1 Change in EDSS scores between 1985 and 2005

\begin{tabular}{|c|c|c|c|c|c|c|c|c|}
\hline \multirow[b]{2}{*}{ EDSS score 1985} & \multicolumn{7}{|c|}{ EDSS score 2005} & \multirow[b]{2}{*}{ Total } \\
\hline & 0-3 & $3.5-5.5$ & $6-7.5$ & $8.0-9.5$ & $\begin{array}{l}\text { MS-related } \\
\text { death }\end{array}$ & Unknown & $\begin{array}{l}\text { Non-MS-related } \\
\text { death }\end{array}$ & \\
\hline $0-3$ & $8(11.4)$ & $10(14.3)$ & $17(24.3)$ & $8(11.4)$ & $10(14.3)$ & $9(12.9)$ & $8(11.4)$ & 70 \\
\hline $6-7.5$ & $1(1.4)$ & $0(0)$ & $5(6.8)$ & $12(16.2)$ & $27(36.5)$ & $6(8.1)$ & $23(31.1)$ & 74 \\
\hline $8.0-9.5$ & $0(0)$ & $0(0)$ & $0(0)$ & $6(8.3)$ & $45(62.5)$ & $0(0)$ & $21(29.2)$ & 72 \\
\hline Unknown & $7(7.8)$ & $6(6.7)$ & $5(5.6)$ & $2(2.2)$ & $27(30.0)$ & $22(24.4)$ & $21(23.3)$ & 90 \\
\hline
\end{tabular}

EDSS, Expanded Disability Status Scale; MS, multiple sclerosis.

Values are expressed with percentage values in parentheses.

Multiple linear regression analysis demonstrated that lower baseline EDSS scores (coefficient $-0.45, \mathrm{p}<0.0001$ ), higher pyramidal functional system score (coefficient $0.38, p=0.02$ ) and a greater number of functional systems involved (coefficient $0.31, p=0.001$ ) were significantly more likely to be associated with greater worsening of disability over 20 years. This analysis did not find disease duration to be an important predictive factor after controlling for baseline EDSS score $(p=0.63)$.

\section{Benign disease}

EDSS scores were available for 289 (76.3\%) with definite or probable MS of the 1985 cohort, of whom 64 (22.1\%) had EDSS $\leqslant 4$ and disease duration of $\geqslant 10$ years. There was no significant difference in the proportion of males and females in the benign and non-benign groups. Those with benign disease had a younger age-at-onset than those in the non-benign group and an increased likelihood of presenting with sensory disturbance in those with benign disease $(p=0.039)$. Ataxia was more common at presentation in those with non-benign disease but this result did not reach statistical significance.

Of the 64 benign cases identified in 1985, 8 (19.0\%) still had an EDSS score of $\leqslant 4$ by 2005, 25 (39.0\%) had an EDSS 4.5-9.5, $9(14.1 \%)$ had died of MS-related causes, 7 (10.9\%) had moved out of the area or were lost to follow-up, and 15 (23.4\%) had died due to non-MS-related causes. Figure 3 shows the EDSS scores in 1985 and 2005 for patients in the benign group. Those patients that died of non-MS-related causes are not shown.

There was no significant difference in terms of age-at-onset, sex, disease duration or symptoms at onset when features at disease onset were compared in those who remained benign 20 years after prevalence day 1985 and those in whom disability increased.

The effects of disease duration and baseline EDSS score were compared to determine if those with lower EDSS scores or longer disease durations were more likely to remain benign after
20 years follow-up (table 3): of those with an EDSS score of 0-2 and a disease duration of $<5$ years, there was a $61.5 \%$ and $53.8 \%$ chance of reaching EDSS 4 and 6 , respectively. This is comparable to $63.6 \%$ and $63.6 \%$ reaching EDSS 4 and 6 , respectively, for those with disease durations of between 10 and 20 years. For those with EDSS scores of between 2.5 and 4, there appeared to be no effect of disease duration on the proportion of patients reaching EDSS 4 and 6.

\section{DISCUSSION}

This study has analysed a large population-based sample of patients and documented change in disability over a 20-year period, demonstrating a mean change of 3.0 EDSS points per patient and 4.1 points per patient for each 20-year period of completed follow-up. Furthermore, the observed mortality rate was $58 \%$, there was a low conversion rate of suspected to probable or definite disease, and high rates of conversion from benign to more intermediate prognostic groups over the longer term. Initial assessment of clinical features was generally found to be poorly predictive of outcome, and the proportion of erroneous diagnoses in those considered at outset to have definite or probable disease was $2.6 \%$, suggesting relatively high specificity of the diagnostic criteria used, although underlining the importance of long-term follow-up.

Direct comparison of long-interval cross-sectional analyses from large MS population-based samples is difficult because too few studies have used comparable methodologies. In particular, it is problematic to compare accurately this work to the wellestablished multi-point data sets from patient cohorts in London, Ontario, Canada, ${ }^{151618} 19$ and Lyon, France, ${ }^{78}$ for these reasons. However, one comparable study from Olmsted county, USA, examined change in disability over a 10-year period in 162 patients finding a mean change of only $+1.0 \mathrm{EDSS}^{12}$ point. More than two-thirds of patients with minimal disability (EDSS <3) were unchanged, of whom only $16.7 \%$ required a stick or had

Table 2 Mean change in EDSS score - significant results

\begin{tabular}{|c|c|c|c|c|}
\hline Factors & $\mathbf{n}$ & Mean change in EDSS & SD & p value \\
\hline Total & 199 & 3.02 & 2.3 & \\
\hline Baseline EDSS score & & & & $<0.0001$ \\
\hline $0-3.5$ & 39 & 4.71 & 2.8 & \\
\hline $4-6$ & 53 & 3.92 & 2.1 & \\
\hline $6.5-8$ & 32 & 2.48 & 2.3 & \\
\hline $8.5-9.5$ & 75 & 1.73 & 1.0 & \\
\hline Disease duration in 1985 & & & & 0.013 \\
\hline$<10$ years & 78 & 3.62 & 2.4 & \\
\hline $10-25$ years & 78 & 2.71 & 2.4 & \\
\hline$>25$ years & 42 & 2.54 & 1.6 & \\
\hline
\end{tabular}

EDSS, Expanded Disability Status Scale; SD, standard deviation. 


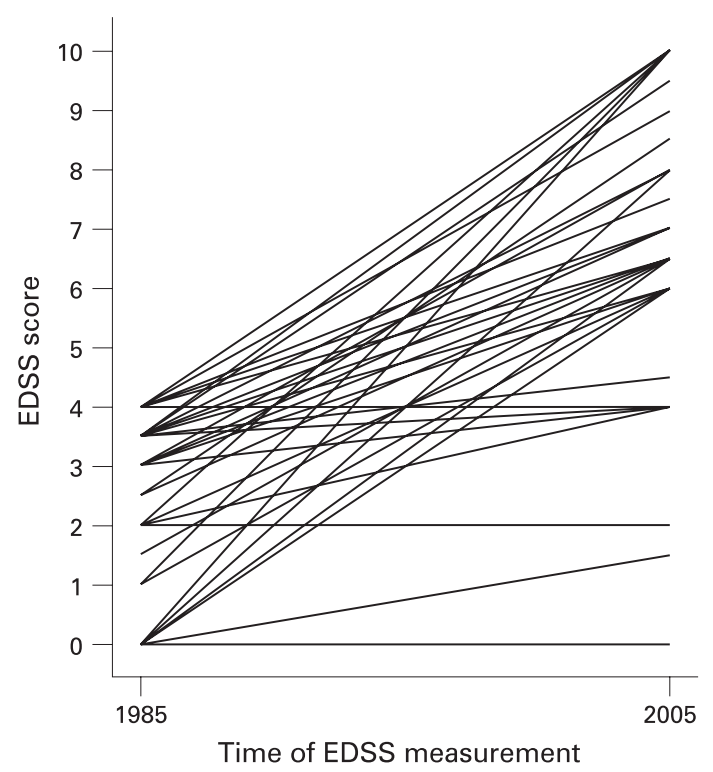

Figure 3 Expanded Disability Status Scale (EDSS) scores in 1985 and 2005 in the benign group.

more severe loss of mobility after 10 years. Furthermore, of those with moderate disability (EDSS scores of 3.5-5), only $51.5 \%$ required the use of a stick or had more severe loss of mobility and only $3 \%$ were bed-bound or wheelchair users after 10 years of follow-up. However, in our study, even patients with little disability (EDSS $\leqslant 3$ ) at the start of the study commonly required assistance to mobilise and more than onethird were bed- or chair-bound or worse after 20 years. Our data also show that, of those with limited mobility but still managing without assistance (EDSS 3.5-5.5), 82\% needed assistance to walk or had more severe loss of mobility (EDSS $\geqslant 6$ ), and $59 \%$ were confined to a bed or chair or had died 20 years later. Our study also suggests that, over 20 years, there were few patients who were ambulatory with assistance on initial assessment who remained stable, and the majority in this group died during the period of observation. Less than $10 \%$ of those who were bed- or chair-bound at initial assessment remained alive. However, it is important to note that patients who had died of unrelated causes did not contribute to data on EDSS change as their EDSS score at death was not known; this could potentially be a significant confounding factor as the registration of cause of death has previously been demonstrated to have limited accuracy.
Being able to predict which patients will rapidly become disabled is of considerable importance with respect to therapeutic choices. Conversely, we have demonstrated that at least 1 in 8 patients will not gain significant disability even after 20 years or more of follow-up, and a similar proportion will not progress regardless of their baseline EDSS score. Such patients are clearly important to identify; however, clinical predictors are rarely robust and we have found few factors that can be reliably used for such a purpose. Lower baseline EDSS scores are associated with a greater degree of worsening; however, this is probably attributable to the non-linear and ordinal nature of the EDSS, particularly with respect to higher levels of disability. Higher pyramidal scores did, however, appear to be associated with greater accumulation of disability as did the greater number of functional systems involved. However, this may be of limited clinical use as these patients will commonly have already gained significant disability before specific treatments can be initiated - in particular, if a secondary progressive disease course has intervened. ${ }^{39}$ In this respect, our survey is not dissimilar to the Olmsted county study, which also found no clinical or demographic factor that strongly predicted outcome. ${ }^{12}$ However, it should also be noted that those with a benign course over the first 10 years of disease did seem more likely to present with sensory signs and symptoms and at a younger age than those with non-benign disease, features also mirrored in two further smaller interval studies. ${ }^{22} 40$

There has long been controversy regarding the group of patients previously classified as having suspected disease in previous epidemiological studies. These individuals have commonly been included in overall prevalence estimates. ${ }^{41}{ }^{42}$ Our study suggests that even those individuals in whom MS was considered likely and classifiable as having MS using earlier diagnostic criteria but not fulfilling the Poser criteria for MS, had a low chance of developing clinically definite or probable disease. This finding has implications for the validity of this group of "suspected" patients in prevalence studies and suggests that they should not be included in epidemiological estimates of disease frequency. The more recent introduction of revisions to the "McDonald Criteria"43 may resolve this issue.

Some observers have suggested that initial disease course may be predictive of final outcome. ${ }^{44}$ In one study, 34 of 49 (69.4\%) patients remained benign after a follow-up of 10 years. ${ }^{45}$ However, our study suggests that only $20 \%$ of patients classified as benign after 10 years or more of disease will continue along this course after a further 20 years of follow-up, and $14.1 \%$ will die of MS-related causes. Our experience is further supported by a study from Ireland showing that only $28.6 \%$ of patients with benign disease continued to fulfil criteria

Table 3 Twenty-year outcome of those with benign disease in 1985 (EDSS score $\leqslant 4$ and disease duration $\geqslant 10$ years) by baseline EDSS score and disease duration

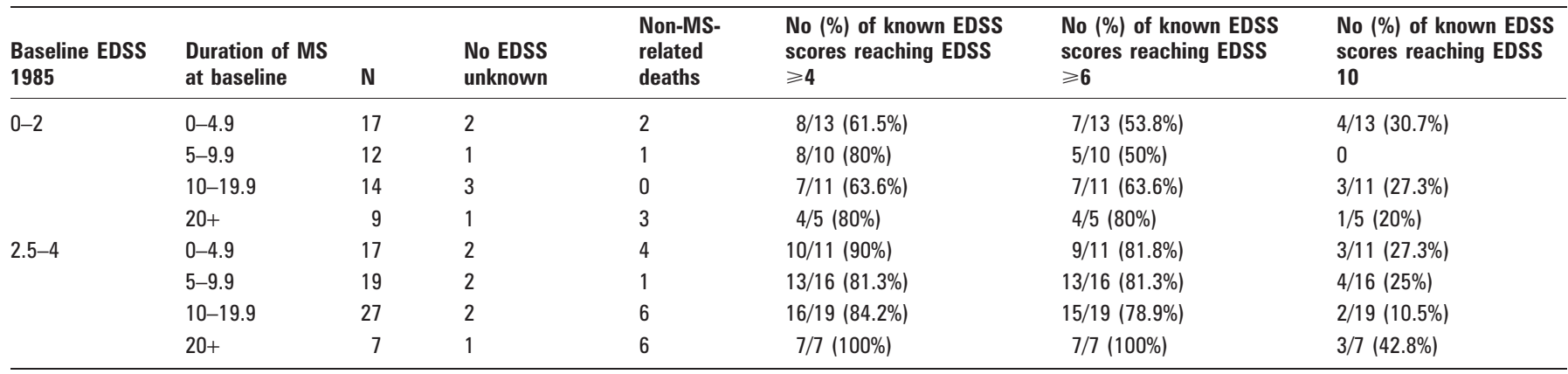


for benign disease after 10 years of follow-up. ${ }^{22}$ Other groups have suggested a more intermediate outcome, with $52.1 \%$ of patients in a large cohort of patients identified from a clinical MS database in Canada continuing to follow a benign course after 10 years of follow-up. ${ }^{46}$

Several studies have also suggested that those with EDSS scores of $\leqslant 2$ at 10 years of disease or more are less likely to progress on follow-up. Sayao et al demonstrated that $32 \%$ of those with an EDSS of $<2$ at $\geqslant 10$ years were no longer benign after 10 years compared to $48 \%$ in those with an EDSS of $<3 .{ }^{46}$ The Olmstead county study suggested that $93 \%$ of those with EDSS scores of $\leqslant 2$ at 10 years remained benign after a further 10 years. ${ }^{45}$ A study from Iceland suggested that, after 30 years from disease onset, $50 \%$ of all patients remain in the benign group defined as EDSS $<4$ after $\geqslant 15$ years. This latter result appears relatively high. However, the authors also suggest that, after 15 years of disease, $70 \%$ of patients fulfil the criteria for benign disease. The reasons for this remain unclear but may relate to regional genetic or environmental factors or, alternatively, to differences in local health care surveillance, which allow the earlier identification of mildly affected cases. ${ }^{20}$ Our study found that, of those with EDSS $0-2$ at $\geqslant 10$ years after 20 years follow-up, 11 of 16 (68.8\%) with known EDSS data have reached EDSS $>6$ and $25 \%$ have died of MS-related causes. Conversely, 23 of 26 (88.4\%) with EDSS $2.5-4$ at $\geqslant 10$ years have progressed to EDSS $>4$ and $84.6 \%$ to EDSS $>6$, and $19 \%$ have died. Although it appears that those with an EDSS of 2.5-4 are more likely to progress over the following 20 years, a high proportion of patients with EDSS 0-2 also progress, suggesting that even those with a low EDSS have a significant chance of gaining moderate to severe disability.

In summary, we have shown that the majority of prevalent patients had worsened by $\geqslant 1$ EDSS point over 20 years. The only factors found to be of use in predicting which patients will have the greatest accumulation increase on the EDSS are those who have lower baseline EDSS scores, higher pyramidal functional system scores and a greater number of functional systems involved. Of those with benign disease in 1985, only $19 \%$ remained benign after 20 years of follow-up but $14 \%$ of patients failed to worsen by $\geqslant 1$ EDSS point. No factors were predictive of remaining in the group with a benign course and even patients with EDSS $\leqslant 2$ were at significant risk of gaining moderate disability with prolonged follow-up. However, it appears that those who would benefit most from early therapeutic interventions would be those aged between 20 and 29 years at disease onset, who have low EDSS scores but relatively high pyramidal functional system scores and who have multi-site involvement.

Acknowledgements: The authors would like to acknowledge Dr Annabel Hennessey for her role in the 1985 prevalence study, the patients who kindly agreed to take part and the Office of Population Censuses and Surveys.

Competing interests: None.

\section{REFERENCES}

1. Bronnum-Hansen H, Koch-Henriksen N, Hyllested K. Survival of patients with multiple sclerosis in Denmark: a nationwide, long-term epidemiologic survey. Neurology 1994;44:1901-7.

2. Poser S, Kurtzke JF, Poser W, et al. Survival in multiple sclerosis. J Clin Epidemiol 1989:42:159-68.

3. Kantarci 0, Siva A, Eraksoy M, et al. Survival and predictors of disability in Turkish MS patients. Turkish Multiple Sclerosis Study Group (TUMSSG). Neurology 1998:51:765-72.

4. Pekmezovic T, Jarebinski M, Drulovic J, et al. Survival of multiple sclerosis patients in the Belgrade population. Neuroepidemiology 2002;21:235-40
5. Amato MP, Ponziani G, Bartolozzi ML, et al. A prospective study on the natural history of multiple sclerosis: clues to the conduct and interpretation of clinical trials. J Neurol Sci 1999;168:96-106.

6. Bronnum-Hansen $\mathbf{H}$, Koch-Henriksen N, Stenager E. Trends in survival and cause of death in Danish patients with multiple sclerosis. Brain 2004;127:844-50.

7. Confavreux C, Aimard G, Devic M. Course and prognosis of multiple sclerosis assessed by the computerized data processing of 349 patients. Brain 1980;103:281-300.

8. Confavreux C, Vukusic S. Accumulation of irreversible disability in multiple sclerosis: from epidemiology to treatment. Clin Neurol Neurosurg 2006;108:327-32.

9. Kremenchutzky M, Rice GP, Baskerville J, et al. The natural history of multiple sclerosis: a geographically based study 9: observations on the progressive phase of the disease. Brain 2006;129:584-94.

10. Phadke JG. Clinical aspects of multiple sclerosis in north-east Scotland with particular reference to its course and prognosis. Brain 1990;113:1597-628.

11. Phadke JG. Survival pattern and cause of death in patients with multiple sclerosis: results from an epidemiological survey in north east Scotland. J Neurol Neurosurg Psychiatry 1987:50:523-31.

12. Pittock SJ, Mayr WT, McClelland RL, et al. Change in MS-related disability in a population-based cohort: a 10-year follow-up study. Neurology 2004;62:51-9.

13. Runmarker B, Andersen 0. Prognostic factors in a multiple sclerosis incidence cohort with twenty-five years of follow-up. Brain 1993;116:117-34.

14. Wallin MT, Page WF, Kurtzke JF. Epidemiology of multiple sclerosis in US veterans. VIII. Long-term survival after onset of multiple sclerosis. Brain 2000;123:1677-87.

15. Weinshenker BG, Bass B, Rice GP, et al. The natural history of multiple sclerosis: a geographically based study. 2. Predictive value of the early clinical course. Brain 1989;112:1419-28.

16. Weinshenker BG, Bass B, Rice GP, et al. The natural history of multiple sclerosis: a geographically based study. I. Clinical course and disability. Brain 1989;112:133-46.

17. Weinshenker BG, Issa M, Baskerville J. Long-term and short-term outcome of multiple sclerosis: a 3-year follow-up study. Arch Neurol 1996;53:353-8.

18. Weinshenker BG, Rice GP, Noseworthy JH, et al. The natural history of multiple sclerosis: a geographically based study. 3. Multivariate analysis of predictive factors and models of outcome. Brain 1991;114:1045-56.

19. Weinshenker BG, Rice GP, Noseworthy JH, et al. The natural history of multiple sclerosis: a geographically based study. 4. Applications to planning and interpretation of clinical therapeutic trials. Brain 1991;114:1057-67.

20. Benedikz J, Stefansson M, Guomundsson J, et al. The natural history of untreated multiple sclerosis in Iceland. A total population-based 50 year prospective study. Clin Neurol Neurosurg 2002;104:208-10.

21. Allison RS. Survival in disseminated sclerosis: a clinical study of a series of cases first seen 20 years ago. Brain 1950; 73:103-20.

22. Hawkins SA, McDonnell GV. Benign multiple sclerosis? Clinical course, long term follow up, and assessment of prognostic factors. J Neurol Neurosurg Psychiatry 1999;67:148-52.

23. Mc Alpine D, Compston N. Some aspects of the natural history of disseminated sclerosis. O J Med 1952;21:135-67.

24. Miller D, Barkhof F, Montalban X, et al. Clinically isolated syndromes suggestive of multiple sclerosis, part I: natural history, pathogenesis, diagnosis, and prognosis. Lancet Neurol 2005;4:281-8.

25. Leibowitz U, Kahana E, Alter M. Survival and death in multiple sclerosis. Brain 1969:92:115-30.

26. Hyllested K. Lethality, duration, and mortality of disseminated sclerosis in Denmark Acta Psychiatr Scand 1961;36:553-64.

27. Myhr KM, Riise T, Vedeler C, et al. Disability and prognosis in multiple sclerosis: demographic and clinical variables important for the ability to walk and awarding of disability pension. Mult Scler 2001;7:59-65.

28. Visscher BR, Liu KS, Clark VA, et al. Onset symptoms as predictors of mortality and disability in multiple sclerosis. Acta Neurol Scand 1984;70:321-8.

29. Bergamaschi R, Montomoli C, Candeloro E, et al. Disability and mortality in a cohort of multiple sclerosis patients: a reappraisal. Neuroepidemiology 2005;25:15-8.

30. Citterio A, Azan G, Bergamaschi R, et al. Multiple sclerosis: disability and mortality in a cohort of clinically diagnosed patients. Neuroepidemiology 1989;8:249-53.

31. Levic ZM, Dujmovic I, Pekmezovic T, et al. Prognostic factors for survival in multiple sclerosis. Mult Scler 1999;5:171-8.

32. Poser S, Poser W, Schlaf G, et al. Prognostic indicators in multiple sclerosis. Acta Neurol Scand 1986;74:387-92.

33. Tremlett H, Paty D, Devonshire V. Disability progression in multiple sclerosis is slower than previously reported. Neurology 2006;66:172-7.

34. Trojano M, Avolio C, Manzari C, et al. Multivariate analysis of predictive factors of multiple sclerosis course with a validated method to assess clinical events. J Neurol Neurosurg Psychiatry 1995;58:300-6.

35. Swingler RJ, Compston DA. The prevalence of multiple sclerosis in south east Wales. J Neurol Neurosurg Psychiatry 1988;51:1520-4.

36. Poser CM, Paty DW, Scheinberg L, et al. New diagnostic criteria for multiple sclerosis: guidelines for research protocols. Ann Neurol 1983;13:227-31.

37. Government Actuary's Department. Historic interim life tables, 2006.

38. Confavreux C, Vukusic S, Moreau T, et al. Relapses and progression of disability in multiple sclerosis. N Engl J Med 2000;343:1430-8.

39. Coles AJ, Cox A, Le Page E, et al. The window of therapeutic opportunity in multiple sclerosis Evidence from monoclonal antibody therapy. J Neurol 2006;253:98-108.

40. Thompson AJ, Hutchinson M, Brazil J, et al. A clinical and laboratory study of benign multiple sclerosis. O J Med 1986:58:69-80. 
41. Roberts MH, Martin JP, McLellan DL, et al. The prevalence of multiple sclerosis in the Southampton and South West Hampshire Health Authority. J Neurol Neurosurg Psychiatry 1991;54:55-9.

42. Mumford CJ, Fraser MB, Wood NW, et al. Multiple sclerosis in the Cambridge health district of east Anglia. J Neurol Neurosurg Psychiatry 1992;55:877-82.

43. Polman CH, Reingold SC, Edan G, et al. Diagnostic criteria for multiple sclerosis: 2005 revisions to the "McDonald Criteria". Ann Neurol 2005;58:840-6.
44. Roxburgh RH, Seaman SR, Masterman T, et al. Multiple Sclerosis Severity Score: using disability and disease duration to rate disease severity. Neurology 2005;64:1144-51.

45. Pittock SJ, McClelland RL, Mayr WT, et al. Clinical implications of benign multiple sclerosis: a 20-year population-based follow-up study. Ann Neurol 2004;56:303-6.

46. Sayao AL, Devonshire V, Tremlett H. Longitudinal follow-up of "benign" multiple sclerosis at 20 years. Neurology 2007;68:496-500.

\section{BMJ Masterclasses}

BMJ Masterclasses are educational meetings designed specifically to meet the learning needs of doctors. They help doctors keep up to date with the latest evidence and recent guidelines in major clinical areas, enabling them to use the latest evidence to make better decisions. The latest evidence, recent guidelines and best practice are delivered in an interactive and informative manner by leading experts. The speakers are specifically chosen as highly-skilled communicators who can authoritatively enthuse the audience and interpret the latest research and guidelines into practical tips for busy doctors. BMJ Masterclasses have proved a huge hit with clinicians, with many saying they have influenced their clinical practice.

http://masterclasses.bmj.com/ 